\title{
Gut bugs, energy balance, and obesity
}

\section{Marc R. Bomhof}

\author{
University of Calgary
}

Obesity and associated chronic disease has seen a dramatic rise in the past 30 years. ${ }^{1}$ Although the etiology of obesity is multifaceted and extremely complex, obesity can be distilled down to positive energy balance. When energy absorbed by the body exceeds energy expended, weight gain is achieved. As the global trend in obesity persists, it is clear that restoring the energy homeostasis equation back to equilibrium remains somewhat of an elusive task. Recently, gut bacteria, or gut microbiota, and the associated short chain fatty acids (SCFA) that they produce have emerged as new players in energy balance and may yield the ability to restore equilibrium. The precise role these bacteria are playing, however, remains somewhat of a mystery.

There are approximately ten times more bacteria cells in the colon than cells in the human body. Comprised of nearly 2000 species and containing around 100 trillion cells, the gut microbiota weighs approximately $1 \mathrm{~kg}$ and yields a genome size 100 times greater than the human genome. ${ }^{2}$ Given the vast 'microbiome' lying in close proximity to the lining of the intestines, microbiota are thought to function as a 'metabolic organ' within the host, providing metabolic and endocrine function, immune system development, and protection against pathogens. Through the process of fermentation, bacteria are able to harness energy from the otherwise indigestible foods entering the colon, producing a range of metabolic by-products such as vitamins, SCFAs (butyrate, propionate, acetate), amino acids, and other odoriferous volatile organic compounds. From a metabolic health and energy balance perspective, these metabolites (in particular SCFA) seem to have a lot of influence.

Research over the past several years has demonstrated the ability of gut microbiota to promote positive energy balance. In studies conducted by Gordon and colleagues, germ free (bacteria free) mice were found to have $40 \%$ less total body fat than mice with a 'normal' gut microbiota.
When the germ free mice were re-inoculated with gut bacteria, total body fat increased by $60 \%{ }^{3}$ Additional studies have found that germ free mice resist weight gain associated with a western diet. ${ }^{4}$ According to the authors, these findings can be attributed to a reduction in SCFA production in the gut and beneficial changes in host geneexpression that limit deposition of fat in adipocytes. Noting that the gut microbiota of lean versus obese individuals are dominated by different bacterial phyla groups, research by Turnbaugh and colleagues established that an obesityassociated gut microbiota was more efficient at extracting energy (SCFA) from feces. ${ }^{5}$ Confirming these findings, a recent human study found that bacterial groups commonly found in obesity are associated with increased energy extraction from stool. ${ }^{6}$

Despite gut microbiota and SCFA being charged with promoting positive energy balance, there is a wealth of evidence that highlights the ability of SCFA-producing bacteria to improve metabolism in favour of a lean phenotype. Supplementation with prebiotic fiber, a nondigestible food ingredient that is highly fermented in the cecael-colon by Bifidobacterium spp., consistently reduces body weight, adipose tissue mass, and consumption of calories. SCFAs, produced from the fermentation of prebiotic fiber, are believed to mediate these effects. Peptide YY, an anorectic hormone that is increased with prebiotic fiber, is reputed to be released from intestinal L-cells after activation of a $\mathrm{G}$ protein-coupled receptor by SCFA. ${ }^{7,8}$ Interestingly, the same $G$ protein-coupled receptors that are found on intestinal L-cells are also found in adipose tissue. SCFA binding to these receptors has been shown to suppress the release of free fatty acids into the blood stream, which can limit the uptake of fat and development of insulin resistance in non-adipose tissues. ${ }^{8}$ Additionally, research by Cani and colleagues has established that bifidogenic prebiotics increase the production of glucagon- 
like peptide-1, a satiety hormone, and glucagon-like peptide 2, a gut trophic hormone that is purported to improve intestinal permeability and reduce systemic inflammation..$^{9,10}$

It is clear that gut microbiota and associated metabolites affect energy balance. The evidence to date has identified that microbiota simultaneously have the ability to influence both sides of the energy balance equation, by increasing energy extraction from food as well as improving the ability to metabolize absorbed energy. What is not clear is the extent and direction to which the collective microbiota, as well as individual bacterial species, are influencing energy balance. Further study is required to determine the 'optimal' energy-balance microbiota. Certainly, with such a vast collection of microbes in the gut, there is a world of metabolic potential to be explored and harnessed. With a growing need for obesity prevention and treatment, gut bacteria research may one day yield novel, minimallyinvasive strategies, utilizing dietary agents such as prebiotics and probiotics, to manage obesity.

\section{References}

1. Lyons R, Raine K, Reading J, Tremblay M, et al. Obesity in Canada. A joint report from the Public Health Agency of Canada and the Canadian Institute for Health Information. 2011.

2. Neish AS. Microbes in gastrointestinal health and disease. Gastroenterology 2009 Jan;136(1):65-80.

3. Backhed F, Ding H, Wang T, Hooper LV, Koh GY, Nagy A, et al. The gut microbiota as an environmental factor that regulates fat storage. Proc Natl Acad Sci U.S.A. 2004 Nov 2;101(44):15718-15723.

4. Backhed F, Manchester JK, Semenkovich CF, Gordon Jl. Mechanisms underlying the resistance to diet-induced obesity in germ-free mice. Proc Natl Acad Sci U.S.A. 2007 Jan 16;104(3):979-984.

5. Turnbaugh PJ, Ley RE, Mahowald MA, Magrini V, Mardis ER, Gordon JI. An obesity-associated gut microbiome with increased capacity for energy harvest. Nature 2006 Dec 21;444(7122):1027-1031.

6. Jumpertz R, Le DS, Turnbaugh PJ, Trinidad C, Bogardus C, Gordon Jl, et al. Energy-balance studies reveal associations between gut microbes, caloric load, and nutrient absorption in humans. Am J Clin Nutr 2011 Jul;94(1):5865.

7. Samuel BS, Shaito A, Motoike T, Rey FE, Backhed F, Manchester JK, et al. Effects of the gut microbiota on host adiposity are modulated by the shortchain fatty-acid binding $\mathrm{G}$ protein-coupled receptor, Gpr41. Proc Natl Acad Sci U.S.A. 2008 Oct 28;105(43):16767-16772.

8. Miyauchi S, Hirasawa A, Ichimura A, Hara T, Tsujimoto G. New frontiers in gut nutrient sensor research: free fatty acid sensing in the gastrointestinal tract. J Pharmacol Sci 2010 Jan;112(1):19-24.

9. Cani PD, Hoste S, Guiot $Y$, Delzenne NM. Dietary non-digestible carbohydrates promote L-cell differentiation in the proximal colon of rats. Br J Nutr 2007 Jul;98(1):32-37.

10. Cani PD, Possemiers S, Van de Wiele T, Guiot Y, Everard A, Rottier O, et al. Changes in gut microbiota control inflammation in obese mice through a mechanism involving GLP-2-driven improvement of gut permeability. Gut 2009 Aug;58(8):1091-1103.

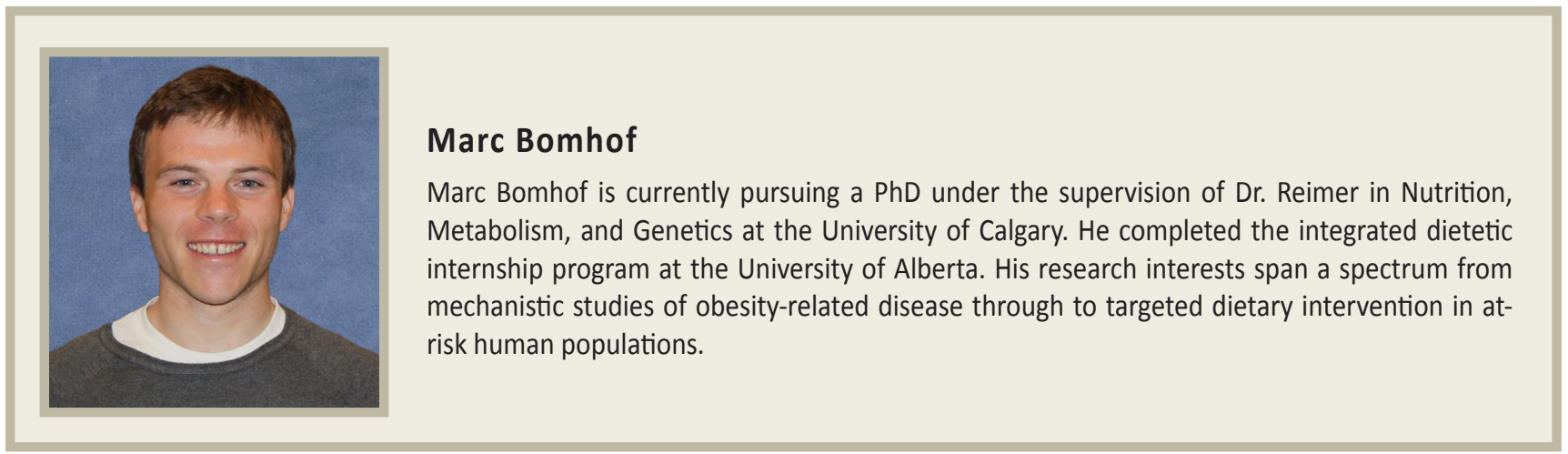

